\title{
Traumatic Neuritis and AFP Surveillance
}

Sir,

National Polio Surveillance Project data is often a subject of continuing debate. ${ }^{1}$ In India, surveillance of Acute Flaccid Paralysis (AFP) is conducted with the use of the Field Guide ${ }^{2}$ in which the diagnosis of Traumatic Neuritis (TN) is ambiguous and imprecise. ${ }^{3}$ The documents used for recording AFP omit details of site and date of injection, which muscles are paralyzed and when. 'Asymmetric' paralysis (is it asymmetric by muscle or limb?) is not defined.

The diagnosis of IN is difficult. ${ }^{4}$ It is significant that the diagnoses of TN appear to be made retrospectively and seem to occur in regions where cases of polio paralysis are frequent. It is common for 1,2 or even 4 or more injections to be given to children with fever and, if that fever has been caused by poliovirus which has reached the spinal cord, aggravation paralysis may result. ${ }^{5}$ About $75 \%$ of polio cases have received injections for fever with paralysis following from 24 to 48 hours later. A small number of cases of provocation polio follow from 6 to 14 days after injections of DPT.

Certainly injections should not be given in the gluteal region, ${ }^{4}$ but far more important is the drastic reduction of any injections except immunizations. Injections for fever are unnecessary and many are unsterile. It is to be regretted that the program for the eradication of

\section{(Author's Reply)}

We appreciate Dr. Mahadevan's thoughtful comments on traumatic neuritis and AFP surveillance, and would like to make a few comments in response.

We agree with Dr. Mahadevan that injections should only be given necessary, and that unnecessary injections can lead to a number of serious health consequences. In addition to the possibility of provocation or aggravation paralysis, unnecessary injections carry an even higher risk of infection with viral diseases. One recent study estimated that up to 39 per cent of all hepatitis $b$ infections were caused by unsafe injections, as well as $40 \%$ of hepatitis $\mathrm{C}$ inections, and at least $5 \%$ of all HIV infections. ${ }^{1}$ Clearly, programs to minimize the use of injections should be promoted.

With respect to surveillance for polio and the case definition of traumatic neuritis (TN), we agree that diagnosing TN on clinical grounds alone may be difficult. In fact, a purely clinical diagnosis of the various potential etiologies of AFP may be very defficult, if not impossible, which has led to the current classification system using poliomyelitis did not include a parallel campaign to reduce the number of injections and, hence, the number of cases of aggravation paralysis.

It is essential that cases of polio are not misdiagnosed as TN.

\author{
S. Mahadevan, \\ Professor, \\ Department of Pediatrics, \\ JIPMER, Pondicherry-605006. \\ E-mail : smahal1232@rediffmail.com
}

\section{REFERENCES}

1. Wenger J, Gupta D, Murthy P. Misinformation regarding national Polio Surveillance project data in Rajasthan. Indian J Pediatr 2003; $70: 683-684$

2. Anonymous. Surveillance of acute flaccid paralysis. Field guide. MCH Division, Department of Family Welfare. Ministry of Health and Family Welfare, New Delhi, India. 2nd edn. $2000 ; 35: 22-23$.

3. Wyatt H V. Diagnosis of acute flaccid paralysis: injection injury or polio? Natl Med J India 2003; $16: 156-158$.

4. Wyatt HV, Mahadevan S, Srinivasan S. Unnecessary Injections and Paralytic Poliomyelitis In India. Trans Roy Soc Trop Med \& Hyg 1992; 86: 546-549.

5. Sharma S, Kale R. Post Injection Palsy in Chhatisgarh Region. Indian Pediatr 2003; 40 : 580-581 both clinical and laboratory (virological) data. The vague definition of TN in the Field Guide, ${ }^{2}$ and failure to report details of site and data of injections was done because of the difficulty in actually using these data for diagnosis and more importantly because the purpose of national AFP surveillance is not to detect TN, but to identify circulating wild polio virus.

If wild polio virus is circulating, it will eventually cause paralysis. By identifying and evaluating all cases of paralysis and evaluating for presence of wild poliovirus, we can locate areas with active wild poliovirus circulation. The current AFP surveillance (used globally as a primary component of the polio eradication initiative), "casts the net widely" and identifies all cases of acute flaccid paralysis in children less than 15 years of age. Cases of TN, Guillain Barre, and other entities, as well as polio, will be included, but that is fine for the purpose of identifying wild poliovirus. From each AFP case, no matter what the etiology, stool specimens are collected in a timely manner, and if wild poliovirus is grown, the case 